\title{
Retrieving Atomic Structure from Dynamical Rocking Curve Measurements in both Real and Reciprocal Space
}

\author{
Christoph T. Koch ${ }^{1}$, Wouter Van den Broek ${ }^{1}$, Feng Wang ${ }^{1}$, Robert S. Pennington ${ }^{1}$ \\ 1. Institute of Physics, Humboldt University of Berlin, Berlin, Germany
}

Fast electrons in a TEM, due to their strong interaction with the electrostatic potential in the material undergo at least some multiple scattering, especially at reduced accelerating voltages. In this presentation, I will show that multiple scattering, often viewed as an annoying side effect that prevents TEM data from being directly interpretable in a fully quantitative manner, may be of great help when solving the crystallographic phase problem, or when retrieving 3D atom positions from HRTEM images of nanoparticles.

The fact that amplitudes and phases of structure factors can be fitted in a highly quantitative manner to the diffraction intensities contained in a single CBED pattern [1] shows us that rocking curve information and multiple scattering in TEM must contain a wealth of information that is waiting to be explored. However, fitting structure factors to CBED patterns of an unknown substance typically requires solving a highly nonlinear system of equations which often precludes obvious strategies to efficiently reach the global minimum of a high-dimensional optimization problem. Large-angle rockingbeam electron diffraction (LARBED) [2] extends the angular range of a conventional CBED experiment. For moderately thick crystals, this extension of the range of incident beam directions up to about $200 \mathrm{mrad}$ allows a straightforward gradient search algorithm to find the correct solution [3] (see Fig. 1).

Although CBED and HRTEM are very different modes of operation of the microscope, the multiple scattering contribution to the signal in tilt series of HRTEM images allows us to retrieve the 3D scattering potential [4], where the position and height of peaks allows direct interpretation as atom species and positions (see Fig. 2). This inversion of multiple scattering is based on an interpretation of the multislice algorithm as an artificial neural network that is taught by feeding it TEM data recorded under different experimental conditions. This can be HRTEM tilt series, ptychography data sets, or scanning confocal electron microscopy (SCEM) data [5].[6]

References:

[1] J.M. Zuo, M. Kim, M. O'Keeffe, and J C.H. Spence, Nature 401 (1999) p. 49.

[2] C.T. Koch, Ultramicroscopy 111 (2011) 828.

[3] F. Wang, R. Pennington, C.T. Koch (2016) submitted.

[4] W. Van den Broek and C.T. Koch, Phys. Rev. Lett. 109 (2012) p. 245502.

[5] W. Van den Broek and C.T. Koch, Phys. Rev. B 87 (2013) 184108

[6] The authors acknowledge funding from the German Research Foundation (DFG) as well as the CarlZeiss Foundation. 

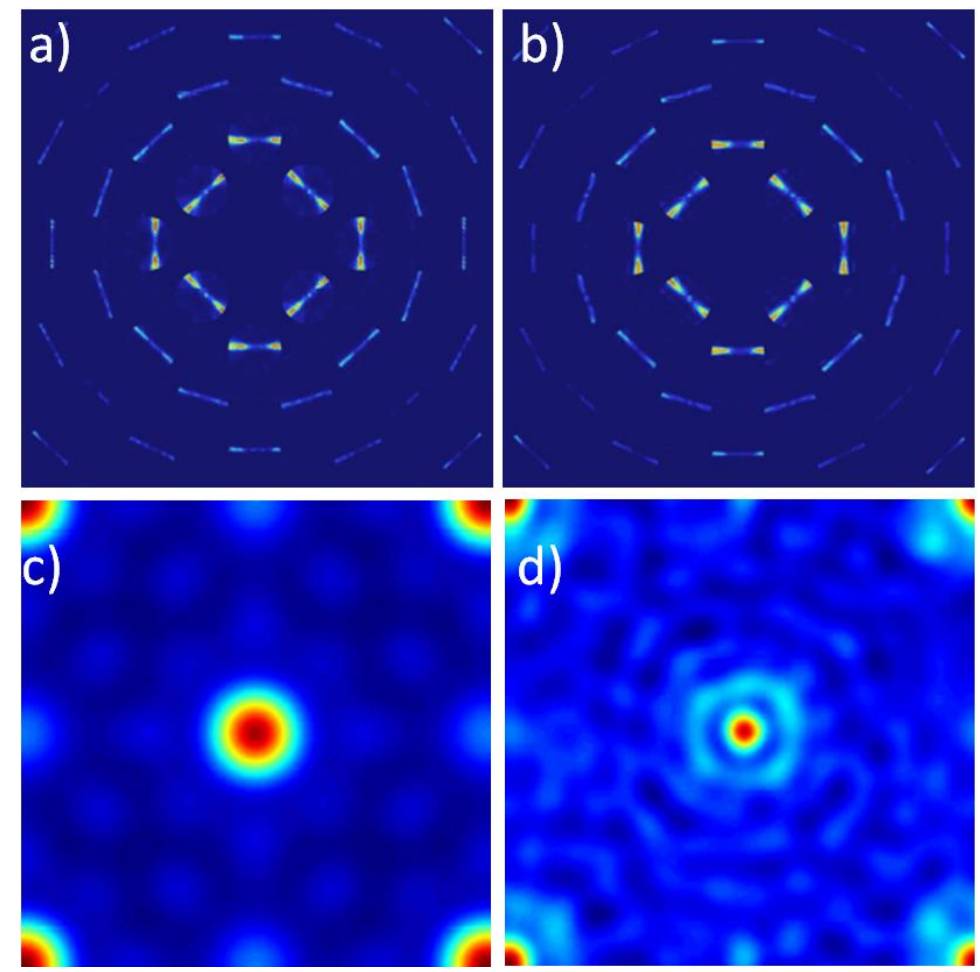

Figure 1: a) Experimental LARBED pattern of $\mathrm{SrTiO}_{3}$ recorded in a Zeiss EM912 without energy filtering (not including the 000 beam). b) Pattern which has been fitted to it, starting with all structure factors initialized to $(0.01+0.01 i) \AA^{-2}$. c) Projected potential of $\mathrm{SrTiO}_{3}(001)$ computed from neutral atom scattering factors, including the first 121 structure factors. d) Projected potential computed from the 456 structure factors fitted to the experimental data shown in (a).
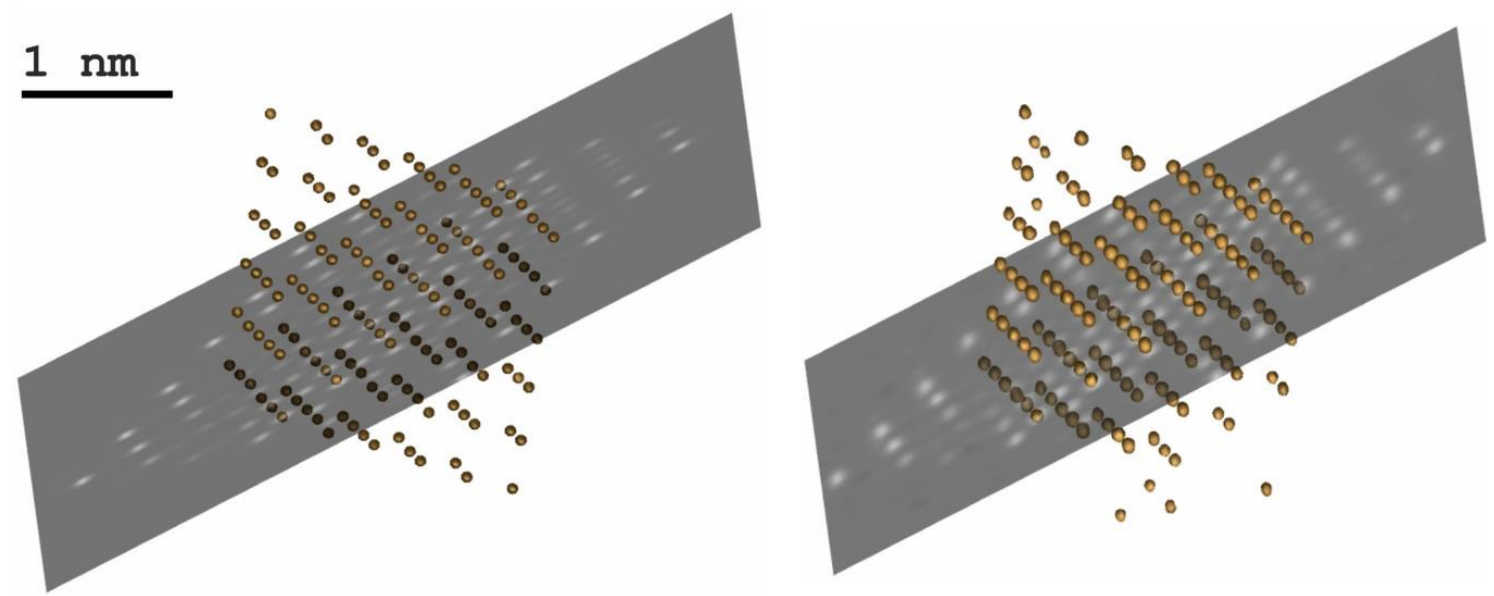

Figure 2: Reconstruction of a PbSe-CdSe core-shell particle with 1963 atoms from a simulated HRTEM tilt series with the alpha-tilt varying from $-10^{\circ}$ to $+10^{\circ}$ in $2^{\circ}$-steps with zero beta-tilt, and the beta-tilt varying from $-10^{\circ}$ to $+10^{\circ}$ in $2^{\circ}$-steps with zero alpha-tilt. The signal-to-noise ratio was 10 , the acceleration voltage $80 \mathrm{kV}$, the defocus $-20 \mathrm{~nm}$, the spherical aberration $64 \mathrm{um}$. Left: Pb-atoms in the original particle's core. Right: The $\mathrm{Pb}$-atoms in the reconstructed potential, identified by simple thresholding, thus demonstrating mass contrast. Due to noise in the measurements, there are some false positives and false negatives. Lowering the threshold would also reveal the $\mathrm{Cd}$ and $\mathrm{Se}$ atoms. 